\title{
TRACKING PLASMA ETCH PROCESS VARIATIONS USING PRINCIPAL COMPONENT ANALYSIS OF OES DATA
}

\author{
Beibei Ma, Seán McLoone and John Ringwood \\ Department of Electronic Engineering, National University of Ireland Maynooth, Maynooth, Ireland \\ beibei.ma@eeng.nuim.ie,sean.mcloone@eeng.nuim.ie,john.ringwood@eeng.nuim.ie
}

\begin{abstract}
Keywords: Semiconductor manufacturing, plasma etching, metal etching, optical emission spectroscopy (OES), principal component analysis (PCA), batch processing.

Abstract: $\quad$ This paper explores the application of principal component analysis (PCA) to the monitoring of within-lot and between-lot plasma variations that occur in a plasma etching chamber used in semiconductor manufacturing, as observed through Optical Emission Spectroscopy (OES) analysis of the chamber exhaust. Using PCA, patterns that are difficult to identify in the 2048-dimension OES data are condensed into a small number of principle components (PCs). It is shown, with the aid of experimental data, that by simply tracking changes in the directions of these PCs both inter-lot and intra-lot patterns can be identified.
\end{abstract}

\section{INTRODUCTION}

Modern day semiconductor manufacturing is a highly competitive business in which companies are required to produce vast quantities of reliable high performance integrated circuits (ICs) at low cost. As such, close monitoring and tight control of hundreds of complex process steps are needed to maintain production standards and high product throughput.

In this context we focus on plasma etching of semiconductor wafers, an important process step in the manufacture of many ICs (Sugawara, 1998). A typical reaction ion etching (RIE) chamber is illustrated in Fig. 1. Gas is pumped into the chamber under vacuum and ionised using a high power Microwave (MF) source to create a plasma. A radio frequency (RF) electromagnetic field accelerates the resulting ionised species towards the electrode, where they interact both chemically and physically with the wafer, etching away the exposed surface. The etch rate and profile obtained are determined in a complex and nonlinear fashion by the plasma chemistry and energy as well as several process variables including gas flow rates and RF power.

Monitoring the chemistry of the plasma in the chamber can be achieved using Optical Emission Spectroscopy (OES) (Splichal et al., 1987). In the plasma chamber considered in this study the OES data is collected for the exhaust plasma leaving the chamber using a 2048 wavelength OES sensor
$(170 \mathrm{~nm}$ to $875 \mathrm{~nm})$ with a sampling interval of $0.75 \mathrm{~s}$. Using this setup OES data was collected for 17 lots of 24 wafers, with each waver undergoing a two step etch process lasting $45 \mathrm{~s}$. A sample OES data set for a single wafer is shown in Fig. 2.

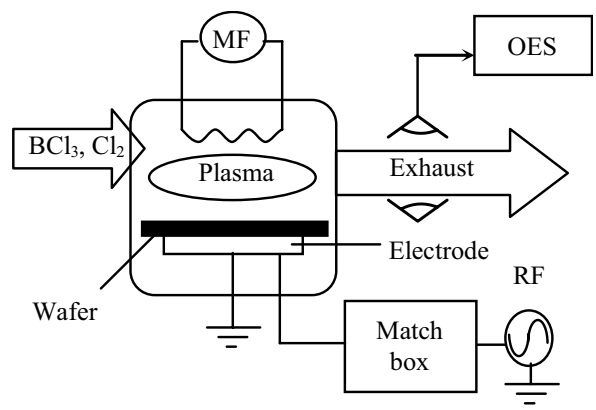

Figure 1: Diagram of a plasma etching chamber.

With the OES footprint of each wafer having dimensions of $60 \times 2048$, direct visualisation and monitoring of variations in the plasma chemistry across wafers and across lots is impractical. Fortunately, optical emission spectra are inherently highly redundant making it possible to achieve substantial data compression using Principle Component Analysis (PCA) techniques without loosing valuable information on plasma changes. In this paper we show that simply monitoring changes 


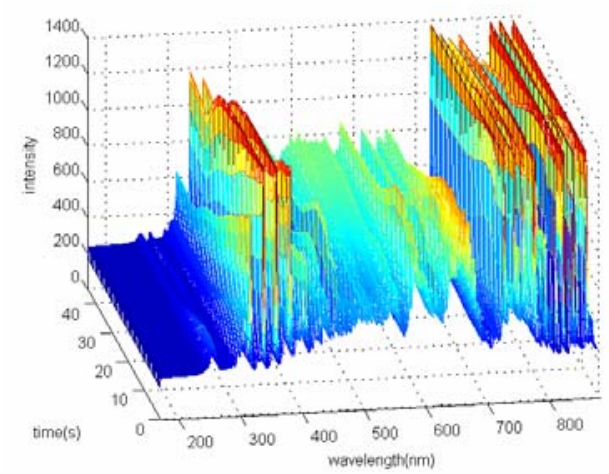

Figure 2: A plasma etch OES data set for a single wafer.

in the directions of the principle component loading vectors, computed on either a wafer-by-wafer or lotby-lot basis, is sufficient to detect valuable information on process trends that are not immediately apparent when looking at the OES data as a whole.

\section{PRINCIPAL COMPONENT ANALYSIS}

Principal component analysis (PCA) is a linear multivariate data projection technique widely used for data compression and analysis (Jackson, 1991). It provides a means of generating low dimension representations of high dimension datasets while retaining the maximum amount of information.

\subsection{Definition}

PCA is a method of writing a matrix $\mathbf{X}$ of rank $r$ as a sum of $r$ matrices of rank 1, where the rank 1 matrices are expressed as outer products of two vectors, a score $\mathbf{t}_{i}$ and a loading $\mathbf{p}_{i}$ (Jackson, 1991)

$$
\mathbf{X}=\sum_{i=1}^{r} \mathbf{t}_{i} \mathbf{p}_{i}^{\mathrm{T}}
$$

The loading vectors, $\mathbf{p}_{i}$, are eigenvectors of the matrix $\mathbf{X}^{\mathrm{T}} \mathbf{X}$, that is

$$
\left(\mathbf{X}^{\mathrm{T}} \mathbf{X}\right) \mathbf{p}_{i}=\lambda_{i} \mathbf{p}_{i}
$$

where $\lambda_{i}$ is the eigenvalue associated with the $i^{\text {th }}$ eigenvector $\mathbf{p}_{i}$. The loading vectors $\mathbf{p}_{i}$ describe the principal directions of variation in $\mathbf{X}$, are orthogonal to each other:

$$
\mathbf{p}_{i}^{\mathrm{T}} \mathbf{p}_{j}=0, \quad \forall i \neq j
$$

and have unit length, while the eigenvalues indicate the amount of variance represented by each direction. For a given $\mathbf{X}$ and $\mathbf{p}_{i}$, the corresponding score vector $\mathbf{t}_{i}$ is given by:

$$
\mathbf{t}_{i}=\mathbf{X} \mathbf{p}_{i}
$$

A principle component (PC) model of $\mathbf{X}$ is then obtained by selecting the components $\left(\mathbf{p}_{i}, \mathbf{t}_{i}\right)$ with the largest eigenvalues to represent it. When data redundancy is high two or three PCs are often sufficient to obtain a good model.

Essentially, PCA projects a high dimensional data space onto a lower dimensional sub-space where the axes are the PC loading vectors and the coordinates of the data the PC score vectors.

Singular Value Decomposition (SVD) can be used to calculate all $r$ principal components in one step. Alternatively, the nonlinear iterative partial least squares (NIPALS) algorithm (Geladi, 1986) can be used to calculate them one at a time in order of significance.

\subsection{Multi-way PCA (MPCA)}

Since batch process data is usually arranged in a 3way matrix (batch-variable-time), it must be unfolded into a 2-way matrix in order to apply PCA. This is known as Multi-way Principal Component Analysis (MPCA) and was first introduced by Wold et al. (1987). There are several ways to unfold a 3way matrix. In this paper, we choose to unfold the data along wavelength direction (Fig. 3), because we are interested in tracking process changes over time.

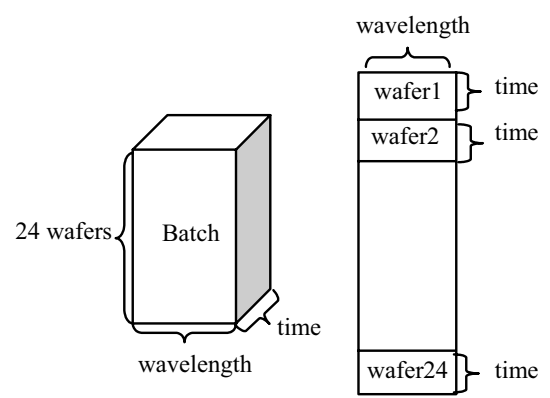

Figure 3: Unfolding of the 3-way OES data blocks. Each block corresponds to a lot of 24 wafers. 


\subsection{Monitoring PC-loadings}

If PCA is performed on the OES data as a whole process trends can only be observed by monitoring the time evolution of the scores. However, if PCA is applied on a wafer-by-wafer or lot-by-lot basis very effective monitoring of process variation can be achieved by tracking the changes in the directions of the PC loadings. Changes can be expressed either in terms the angle between loadings or the magnitude of the vector difference between them as illustrated in Fig. 4. The angle $\theta$ (in radians) is given by

$$
\theta=\arccos \left(\frac{\mathbf{v}_{1} \mathbf{v}_{2}^{\mathrm{T}}}{\left|\mathbf{v}_{1}\right|\left|\mathbf{v}_{2}\right|}\right)
$$

while the magnitude of the vector difference $\varphi$ is simply defined as

$$
\varphi=|\Delta \mathbf{v}|=\left|\mathbf{v}_{1}-\mathbf{v}_{2}\right|
$$

Since, by definition, loading vectors are unit length it follows that for small $\theta$ the two measures are approximately equivalent, i.e. $\varphi \approx \theta$.

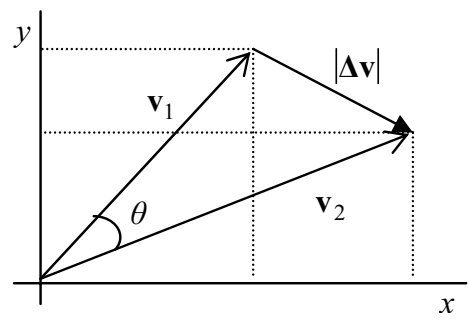

Figure 4: Measuring changes in loading vector directions.

\section{OES DATA ANALYSIS}

\subsection{Data Pre-processing}

Data pre-processing is an essential first step in PCA analysis. Variables need to be appropriately scaled and irrelevant or corrupted measurements removed if valid and interpretable results are to be obtained. In this study the following pre-processing step were performed on the OES data: (1) Data segments corresponding to non-etch periods at the start and end of each etch cycle were removed; (2) Saturated wavelengths were omitted and; (3) Wavelength intensities were scaled to have zero mean.

\subsection{Lot-by-lot Analysis}

Having unfolded the OES data as indicated in Fig. 3, analysis by PCA can be performed by treating each lot of 24 wafers as a single data matrix. We will refer to the resulting PCs as lot-PCs, consisting of lot-PC loadings and lot-PC scores. The variance explained by the first three lot-PCs is plotted as a function of lot number in Fig. 5. This shows that across all lots the first three principal components can explain over $99 \%$ of the plasma variation captured by the OES data. In fact the first PC captures over $85 \%$ of the data variation observed across all 2048 wavelengths.

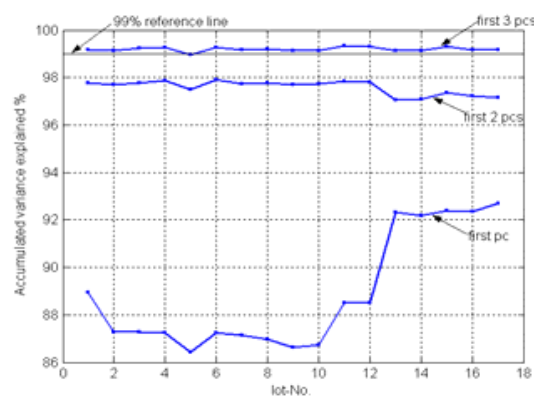

Figure 5: Accumulated variance explained by the first three lot PCs.

A closer look at Fig. 5 shows that a jump occurs in the variance explained by lot- $\mathrm{PC}_{1}$ at lot 13 . Analysis of the variation in the direction of lot- $\mathrm{PC}_{1}$ across lots (Fig. 6) reveals that this is linked to a significant change in the orientation of lot- $\mathrm{PC}_{1}$ from lot 13 onwards. Following investigation it was determined that the plasma change was as a result of a small drift in the flow rate of a cooling gas applied to the backside of the wafers during etching, a change that was not detected by the existing plasma chamber process monitoring schemes.

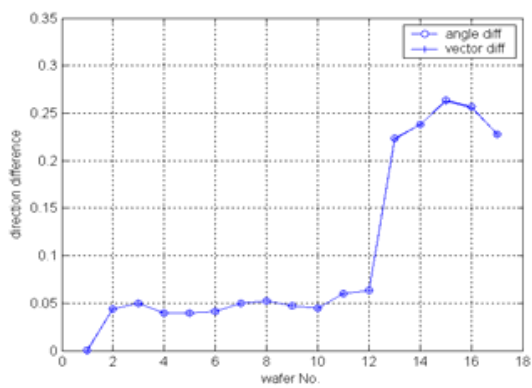

Figure 6: Variation in lot- $\mathrm{PC}_{1}$ (loading) direction across lots (with respect to lot 1 ). 


\subsection{Wafer-by-wafer Analysis}

Here, we simply perform PCA analysis on individual wafer OES data sets and compare the patterns across wafers. This allows us to explore the variation that takes place within lots.

Fig. 7 shows the variation in wafer- $\mathrm{PC}_{1}$ direction over all the wafers with lot- $\mathrm{PC}_{1}$ from lot 1 taken as a reference. The plasma change at lot 12 observed in the lot-PC analysis is clearly present in this data as well, as is a small offset during the first lot.

Large spikes are evident throughout Fig. 7. These occur at the first wafer in each lot. This is highlighted in Fig. 8 which shows a zoomed in view of Fig. 7 covering two lots. These sharp changes were attributed to changes in the absorption characteristics of the plasma chamber wall as a result of a cleaning cycle that is performed between lots. While a dummy etch cycle is performed following each clean cycle to counter this affect, it is clear from Fig. 8 that cleaning still has a significant impact on plasma characteristics for the first (and to a lesser extent) the second wafer etch of each lot.

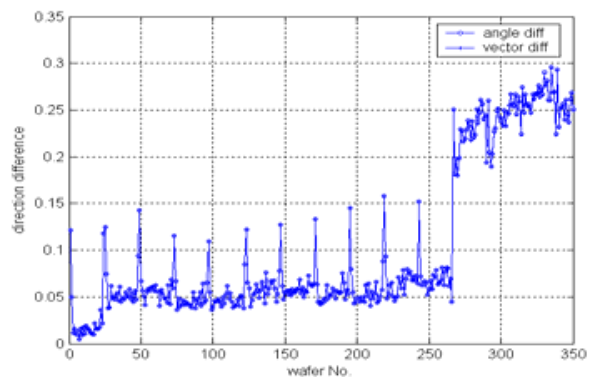

Figure 7: Variation in wafer- $\mathrm{PC}_{1}$ direction across all wafers (using lot- $\mathrm{PC}_{1}$ from lot 1 as a reference).

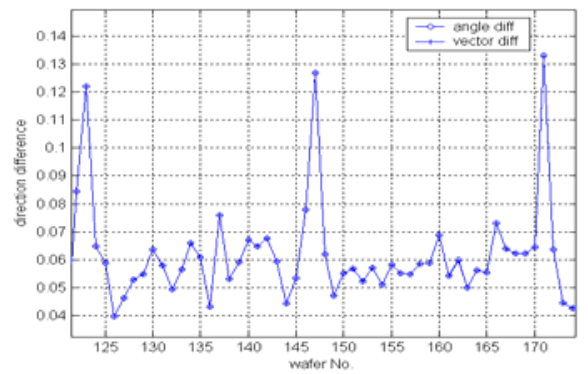

Figure 8: Variation in wafer- $\mathrm{PC}_{1}$ direction over two lots.

\subsection{Score Patterns}

As an illustration of the data compression and pattern visualisation capabilities of PCA the score patterns generated by the OES data for all the wafers in lot 9 are plotted in Fig. 9. Here, the first three lotPC loadings from lot 8 were used as a reference PC model and the PC-scores for each wafer calculated according to Eq. (4). It is easy to see that the evolution of the OES data for the first and second wafers is substantially different from the remaining wafers, as predicted by the wafer-PC loading analysis.

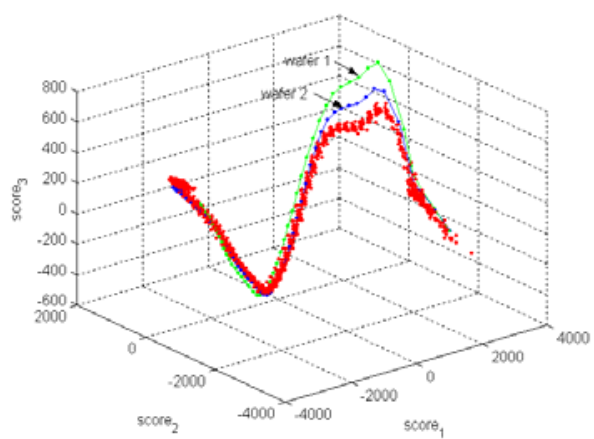

Figure 9: The first three scores of all the wafers in lot 9.

\section{CONCLUSIONS}

In this paper we have demonstrated how monitoring changes in PC directions can be a useful tool in revealing patterns contained in the high dimensional data sets generated from OES analysis of wafer etch plasma chambers.

\section{ACKNOWLEDGEMENTS}

The financial support provided by Enterprise Ireland is gratefully acknowledged.

\section{REFERENCES}

Sugawara, M., 1998. Plasma Etching: Fundamentals and applications, Oxford University Press, New York.

Splichal, M., Anderson, H., 1987. Application of Chemometrics to Optical Emission Spectroscopy for Plasma Monitoring. Proc. SPIE, 2, pp. 189-203.

Jackson, J. E., 1991. A User's Guide to Principal Components, Wiley Interscience Press, New York.

Geladi, P., Kowalski, R. B., 1986. Partial least-squares regression: a tutorial. Analytica Chimica. Acta., 185, pp.1-17.

Wold, S., Geladi, P., Esbensen, K., Ohman, J., 1987. Multi-way principal components and PLS analysis. Journal of Chemometrics, 1, pp. 41-56. 